\title{
Millimole per Liter per Kilogram per Meter Squared
}

National Cancer Institute

\section{Source}

National Cancer Institute. Millimole per Liter per Kilogram per Meter Squared. NCI

Thesaurus. Code C119412.

A unit of concentration equal to millimoles per liter, divided by kilograms per meter squared. 